\title{
A novel surgical approach with cervical preservation for pelvic organ prolapse with uterine descent: The TAP-GAN technique
}

\author{
omer tapisiz ${ }^{1}$, Ali Dogan ${ }^{1}$, and sadiman kiykac altinbas ${ }^{2}$ \\ ${ }^{1}$ University Of Health Sciences, Etlik Zubeyde Hanim Women's Health Training and \\ Research Hospital \\ ${ }^{2}$ Etlik Zubeyde Hanim Kadin Hastaliklari Egitim ve Arastirma Hastanesi
}

May 14, 2020

\begin{abstract}
The lifetime risk for prolapse surgery is nearly $20 \%$, and worldwide vaginal hysterectomy is the most commonly performed surgical procedure for uterine prolapse. Uterine-sparing procedures are attractive options in women without contraindications for these surgeries. The majority of these procedures involve mesh-based approaches; however, patients and surgeons are increasingly worried about the use of meshes. For a meshless urogynecological perspective, we aim to introduce a novel surgical technique in which the critical organ cervix, serving as a roof in terms of pelvic-support, is isolated, after which the prolapse of the remaining uterus is removed, and all pelvic compartments are revised.
\end{abstract}

\section{Introduction}

Pelvic organ prolapse (POP) is a common event that affects $41-50 \%$ of women. ${ }^{1}$ The lifetime risk for prolapse surgery is nearly $20 \%,{ }^{2}$ and worldwide vaginal hysterectomy (VH) is the most commonly performed surgical procedure for uterine prolapse. On the other hand, some authors have recently supported the view that uterine-sparing procedures are attractive options in women without contraindications for these surgeries. ${ }^{3,4}$ The majority of these procedures involve mesh-based approaches; however, patients and surgeons are increasingly worried about the use of meshes due to recent warnings by the UK and the Food and Drug Administration (FDA) ${ }^{5-7}$ Surgeons are searching evidence-based, efficient, safer, and robust native tissue options for their patients.

For a meshless urogynecological perspective, we aim to introduce a novel surgical technique in which the critical organ cervix, serving as a roof in terms of pelvic support, is isolated, after which the prolapse of the remaining uterus is removed, and all pelvic compartments are revised. The crucial point of our procedure is the preservation of the uterine cervix and its ligaments, which play a vital role in the pelvic supporting structures, and the termination of all supporting repairs on the cervix and its ligaments in the apical compartment (Video S1) .

\section{Case and Surgical Technique}

A 67-year-old patient with eight previous vaginal births, and who has been in menopause for 15 years, was admitted to the urogynaecology department of a tertiary research and education hospital in Ankara, complaing of a bulging vaginal mass. In the preoperative examination, using the Pelvic Organ Prolapse Quantification System, stage 3 uterine descent with elongatio colli, stage 3 cystocele, stage 3 rectoenterocele were identified (Aa: +2 , Ba: +2, Ap: +3 , Bp: +4, gh: 6, pb: 3, C: +6 , D: -2.5 , tvl: 8) (Figure S1a) .

The procedure was performed via the vaginal route in the dorsal lithotomy position, and the operation began as a traditional $\mathrm{VH}$. 
In the first step, the cervical length was carefully reviewed to protect the $1.5-2 \mathrm{~cm}$ portion of the cervical tissue, and a circumferential incision was performed around the cervix. With the uterus on upward and lateral retraction via the Jacobs tenacula on the cervix, the uterosacral and cardinal ligament complexes were clamped adjacent to the cervix, incised, and ligated with a 0 delayed-absorbable suture. In this step, the surgeon should pay more attention to preserving the 1.5-2 cm cervix and its supportive tissue of the remaining uterosacral and cardinal ligaments.

In the second step, with the help of an anteriorly placed Deaver retractor, the bladder and the anterior vaginal wall were lifted superiorly for safe localization of the anterior peritoneum. A similar technique was applied to detach the posterior vaginal wall from the posterior cervix and posterior uterine wall. With upward traction applied to the cervix, the peritoneum of the posterior cul-de-sac was then incised with a sharp-curved Mayo scissors, and the pouch of Douglas was opened. A finger was then placed in the posterior cul-de-sac directed towards the anterior cul-de-sac for the identification of the vesicouterine fold of the peritoneum. The peritoneum was then grasped and incised with a pair of sharp-curved Mayo scissors under manuel guidance.

In the third step, the cervix was retracted downward in the midline via the Jacobs tenacula on the cervix, and the anterior uterine wall was grasped with the Backhaus clamps. Then, the surgeon delivered the uterine fundus anteriorly with a hand-over-hand "walking out " technique. Afterwards, the uteroovarian, round, and broad ligaments were clamped from top to bottom with two Heaney clamps and sharply divided medially to the clamp near the uterine fundus until the $1.5-2 \mathrm{~cm}$ of desired cervical tissue to be preserved was reached (advanced energy modalities can also be used). During this step, the surgeon should pay careful attention to maintaining blood supply to the cervical tissue that is desired to be preserved.

In the fourth step, uterine segments at the top (uterine fundus/corpus) and bottom of the cervical tissue were divided using electrocautery, which was also used to cauterize and destroy the endocervix of the preserved part. Afterwards, the modified external McCall culdoplasty was performed by placing sutures on the peritoneum, the preserved cervix, and its supportive ligaments (native tissue).

In the fifth step, anterior and posterior colporrhaphy and enterocele repairs were performed by affixing the final sutures to the preserved cervix and its supporting ligaments. This step, wherein apical support was provided to the preserved cervix and its ligaments (native tissue), was a critical point of our technique.

The operation was completed in 90 minutes (Figure S1b). The patient was discharged uneventfully two days post-operation. At the 6-month follow-up, pelvic examination revealed normal anatomical position (Aa: -3, Ba: -3, Ap: -3, Bp: -3, gh: 3.5, pb: 4.5, C: -7 , D: -7, tvl: 7) and absence of unusual symptoms or adverse events.

\section{Discussion}

The takeaway message is that the cervix, including its ligaments, is an essential structure for pelvic stability. The cardinal ligaments that connect the cervix to the lateral pelvic wall and the uterosacral ligaments that extend posteriorly from the cervix to the sacrum play an essential role in pelvic support that helps to prevent POP(Figure S2a). In our TAP-GAN technique, the $1.5-2 \mathrm{~cm}$ cervical tissue and its ligaments are protected, and all repairs during the POP surgery are attached to this strong component in the apical segment. We expect that this anatomically preserved key organ-cervix will prove advantageous over current alternatives by proactively supporting the natural anatomy during POP surgery.

Among women undergoing hysterectomy for benign reasons, the incidence of occult uterine malignancy ranges from $0.09 \%$ to $3.17 \% .{ }^{8-10}$ Desai et al. reported the prevalence of occult corpus uteri, cervical, and ovarian malignancy to be $1.44 \%, 0.60 \%$, and $0.19 \%$, respectively, among women undergoing hysterectomy for benign indications. ${ }^{9}$ In another study, Frick et al. assessed the risk of unanticipated abnormal gynaecologic pathology at the time of hysterectomy for uterovaginal prolapse, concluding that the risk of unanticipated uterine pathology was $2.6 \%$ in postmenopausal women without bleeding. ${ }^{10}$ Due to these unexpected malignancy rates, the presented technique, in which the cervix is preserved exclusively, is one step ahead according 
to other procedures in which the uterus is preserved as a whole. However, it should be noted that some of the cervix remains after employing this technique. At this point, to be considered is that of regular medically confirmed normal PAP smear results before performing our technique. Additionally, It will be more appropriate to perform this procedure for patients over 65 years of age, where the cervical cancer screening program ends, until we see our short and long term results. On the other hand, removing the entire ectocervix and destroying the endocervix with electrocautery, as in our technique, both considerably reduce the risk of developing cancer.

In conclusion, the TAP-GAN technique is a novel surgical approach with cervical preservation for POP with uterine descent. This approach will be published for the first time in the literature. It should be mentioned that a robust, natural apical support will be provided by preserving the cervix and its supportive ligaments, which play a critical role in pelvic support, rendering anatomical support complete and flawless, with a cervix that looks just like an eagle spreading its wings (Figure S2b) .

\section{Ethics approval and informed consent agreement}

An ethics board approval for the study was obtained from Institutional Review Board (Department of Gynecology/Urogynecology, University of Health Sciences, Etlik Zubeyde Hanim Women's Health Training and Resaerch Hospital, Ankara, Turkey) with the reference number 20092019-02. The patient signed an informed consent that allows us to use her data.

\section{References}

1. Barber MD, Maher C. Epidemiology and outcome assessment of pelvic organ prolapse. Int Urogynecol J 2013; 24(11):1783-90. doi: 10.1007/s00192-013-2169-9

2. Smith FJ, Holman CD, Moorin RE, Tsokos N. Lifetime risk of undergoing surgery for pelvic organ prolapse. Obstet Gynecol 2010; 116(5):1096-100. doi: 10.1097/AOG.0b013e3181f73729

3. Gutman R, Maher C. Uterine-preserving POP surgery. Int Urogynecol J 2013; 24(11):1803-13. doi: 10.1007/s00192-013-2171-2

4. Ridgeway BM. Does prolapse equal hysterectomy? The role of uterine conservation in women with uterovaginal prolapse. Am J Obstet Gynecol 2015;213:802-9.

5. Urinary incontinence and pelvic organ prolapse in women: management. NICE guideline. National Institute for Health and Care Excellence. [Internet] April 2019 Available from: http://www.nice.org.uk/guidance/ng123/resources/urinary-incontinence-and-pelvic-organprolapse-inwomen-management-pdf-66141657205189 (Accessed on April 01, 2020).

6. FDA Strengthens Requirements for Surgical Mesh for the Transvaginal Repair of Pelvic Organ Prolapse to Address Safety Risks. FDA News Release. [Internet] 2016. Available from: http://www.fda.gov/newsevents/newsroom/pressannouncements/ucm479732.htm (Accessed on May 11, 2020).

7. Urogynecologic Surgical Mesh Implants. U.S. FDA. [Internet] April 2019. Available from: http://www.fda.gov/MedicalDevices/ProductsandMedicalProcedures/ImplantsandProsthetics/UroGynSurgicalMesh/c (Accessed on May 11, 2020).

8. Mahnert N, Morgan D, Campbell D, Johnston C, As-Sanie S. Unexpected gynecologic malignancy diagnosed after hysterectomy performed for benign indications. Obstet Gynecol 2015; 125(2):397-405. doi: 10.1097/AOG.0000000000000642

9. Desai VB, Wright JD, Schwartz PE, Jorgensen EM, Fan L, Litkouhi B et al. Occult Gynecologic Cancer in Women Undergoing Hysterectomy or Myomectomy for Benign Indications. Obstet Gynecol 2018; 131(4):642-651. doi: 10.1097/AOG.0000000000002521

10. Frick AC, Walters MD, Larkin KS, Barber MD. Risk of unanticipated abnormal gynecologic pathology at the time of hysterectomy for uterovaginal prolapse. Am J Obstet Gynecol. 2010; 202(5):507.e1-4. doi: 10.1016/j.ajog.2010.01.077

\section{Disclosure of interest}

The authors declare no conflict of interest. 


\section{Contribution to authorship}

Omer Lutfi Tapisiz; Surgeon, conception and design of the operation, drafting of manuscript, last edition of the manuscript.

Ali Riza Dogan; Surgeon, conception and design of the operation, last edition of the manuscript.

Sadiman Kiykac Altinbas; Surgeon, conception and design of the operation, drafting of manuscript, edit of the video, last edition of the manuscript.

\section{Funding}

None

Figure S1a. The preoperative view of the patient's pelvic organ prolapse

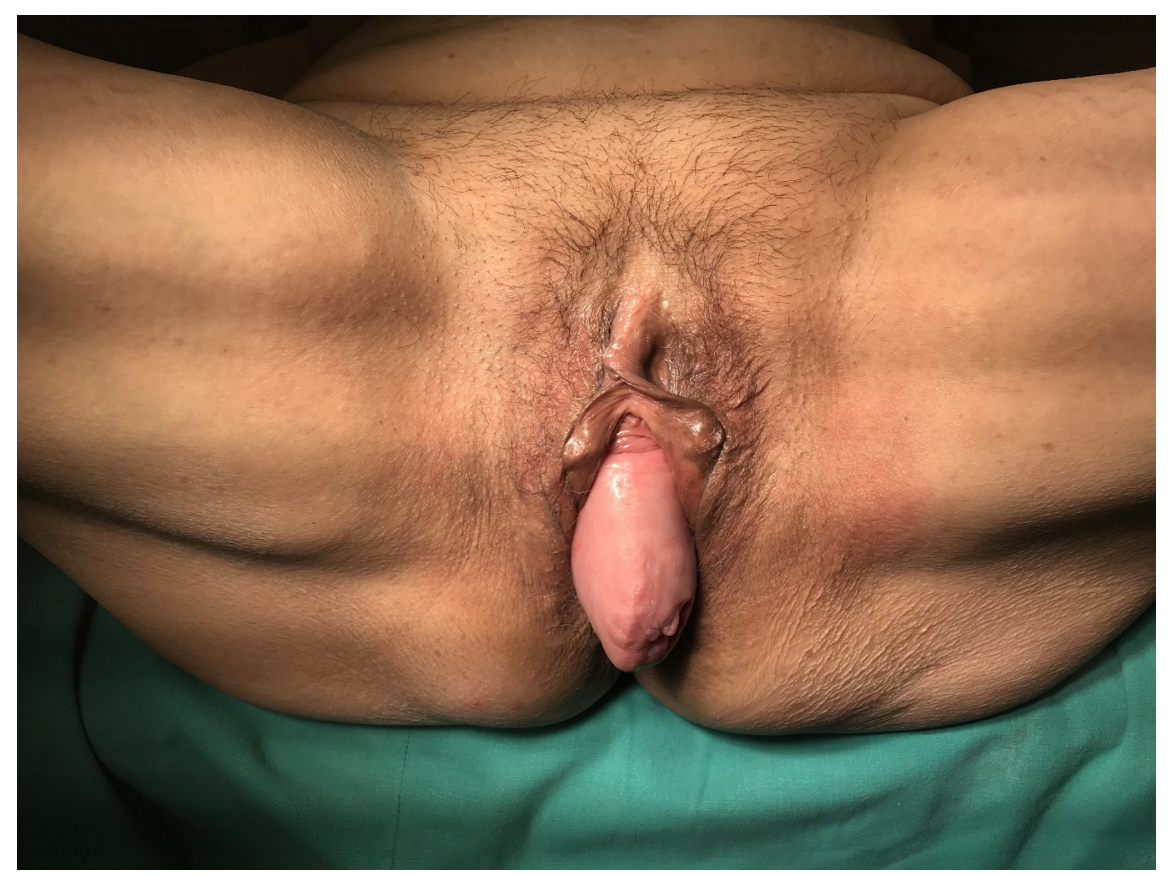

Figure S1b. The postoperative view of the patient's perineum 


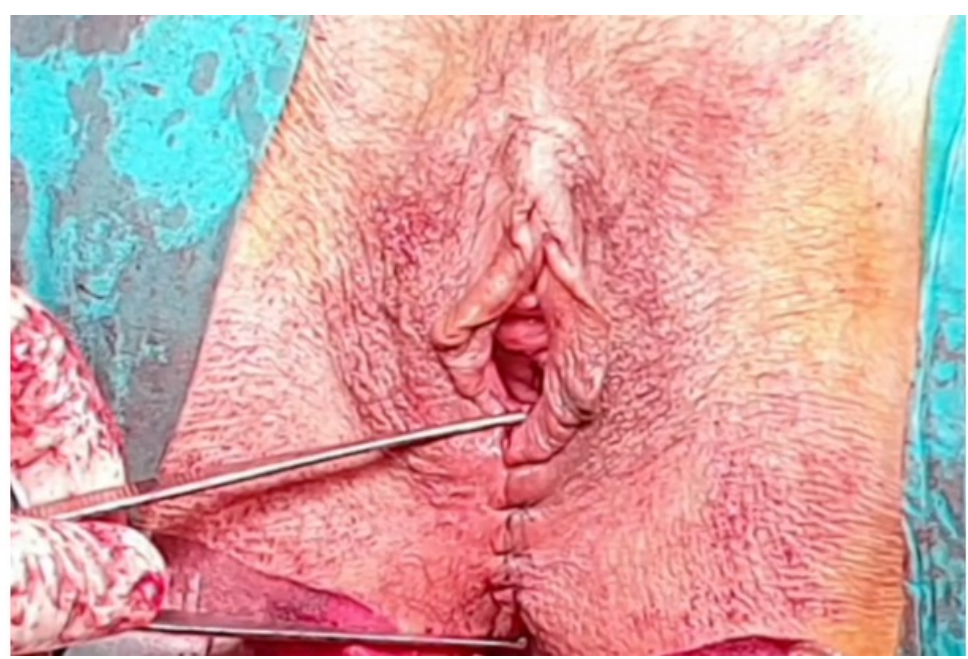

Figure S2a. The illustration of the cervix and its supportive ligaments

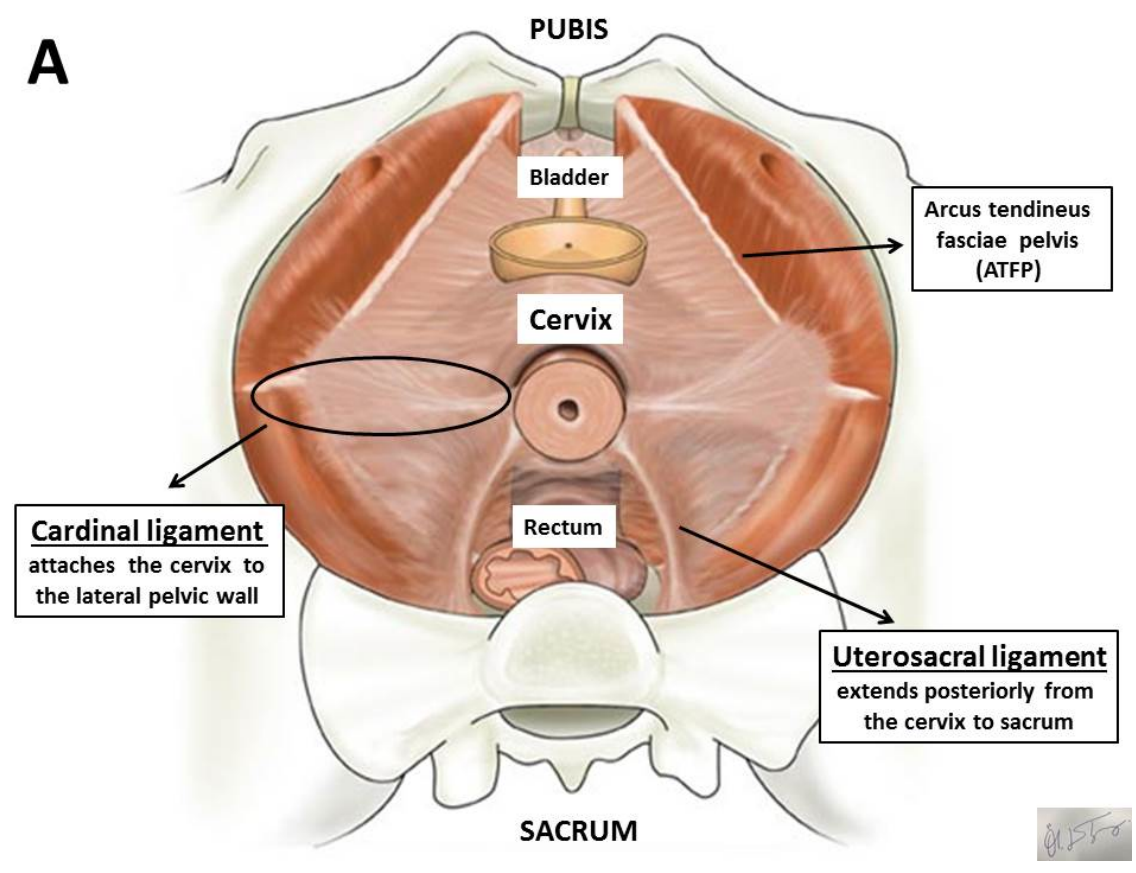

Figure S2b. The illustration of the complete and flawless anatomical support with a cervix that looks like an eagle spreading its wings 


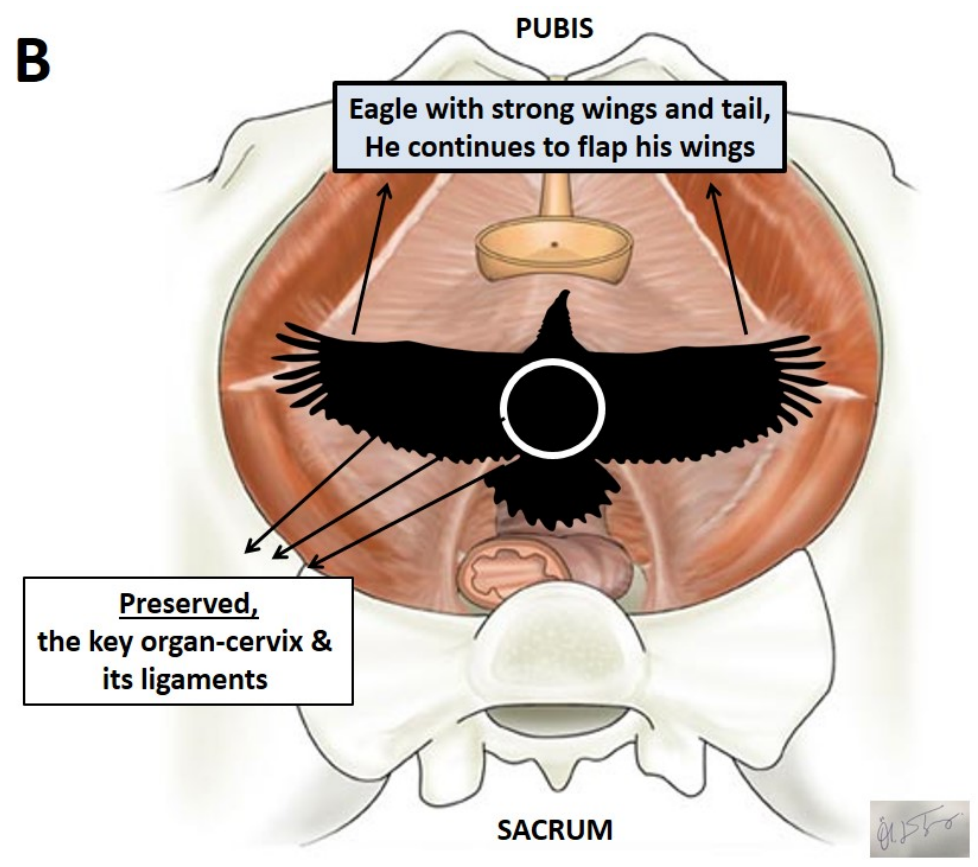

Video S1. Step-by-step video explanation of TAP-GAN technique.

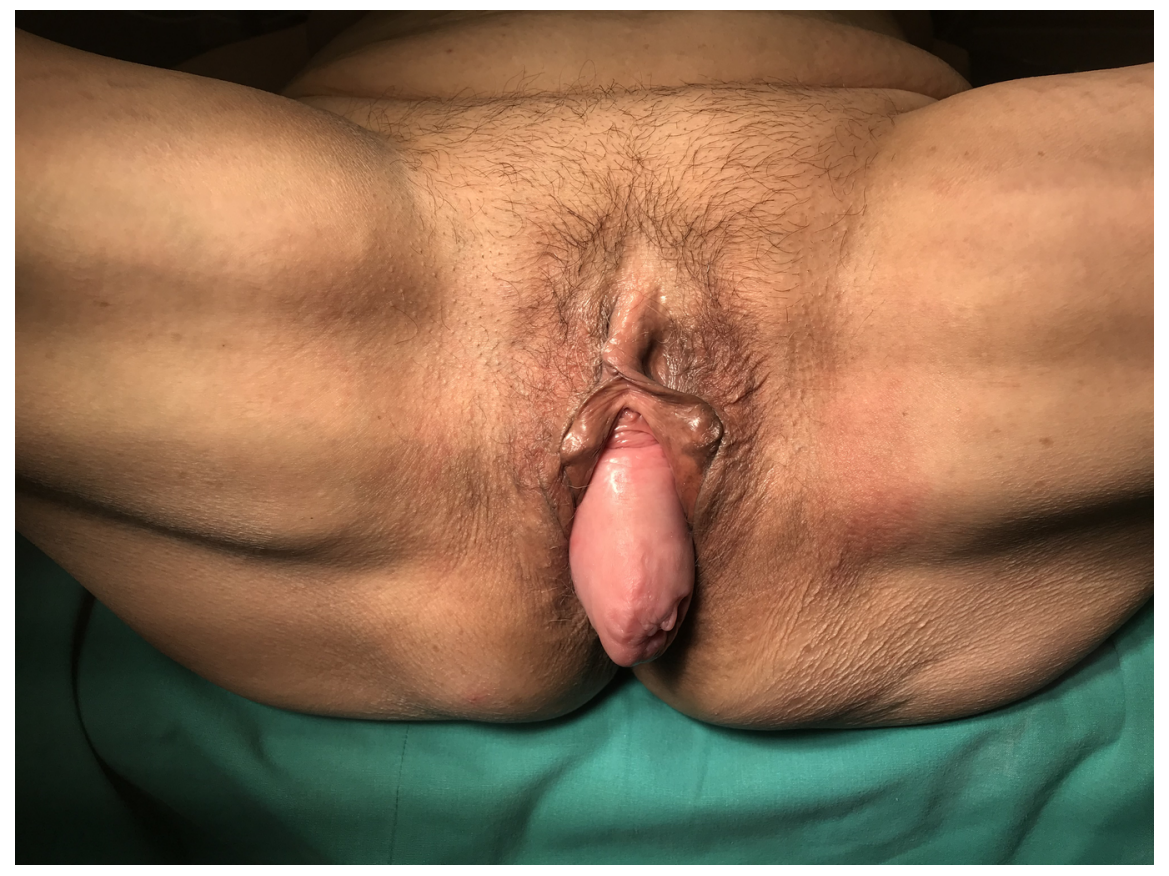



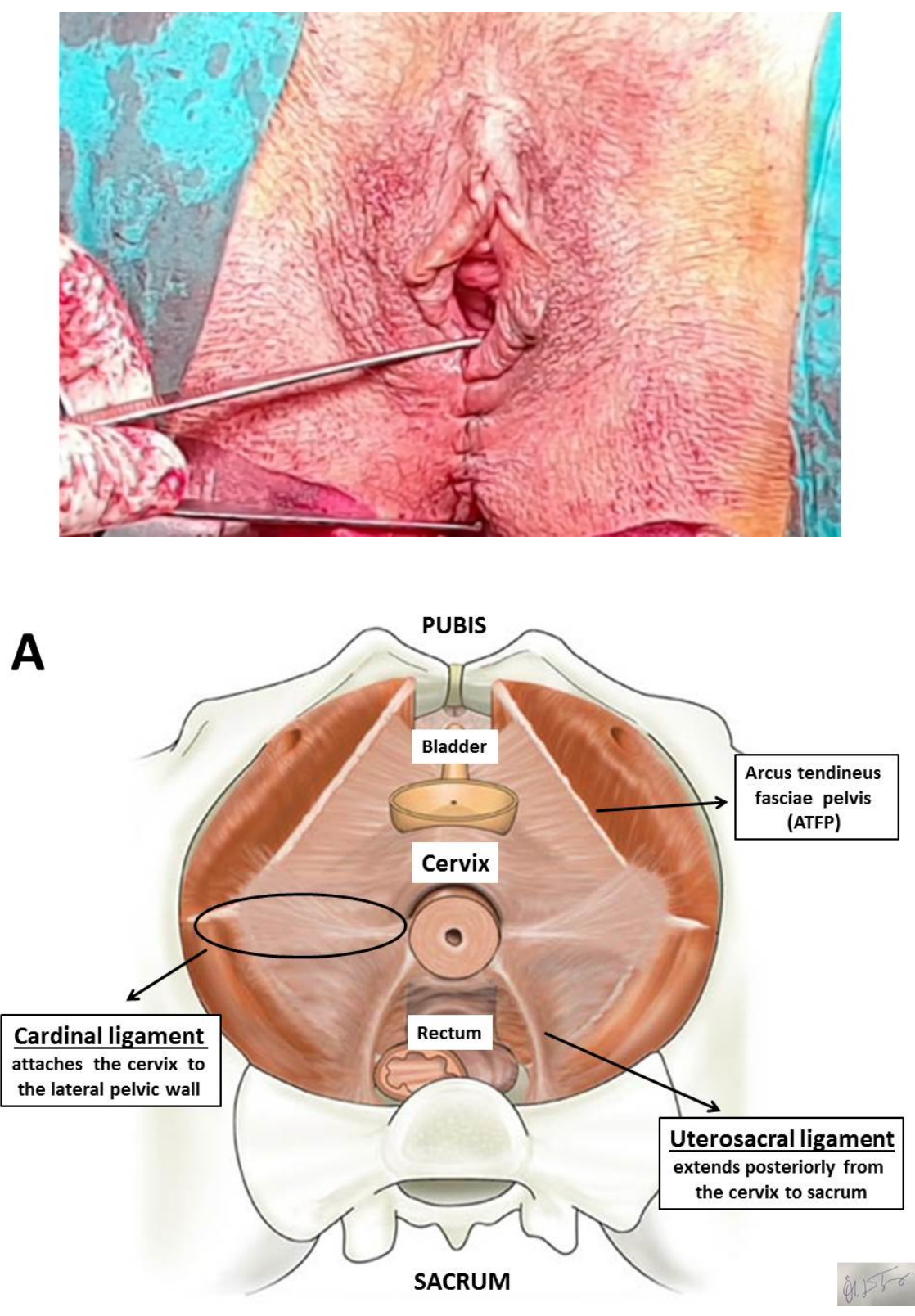


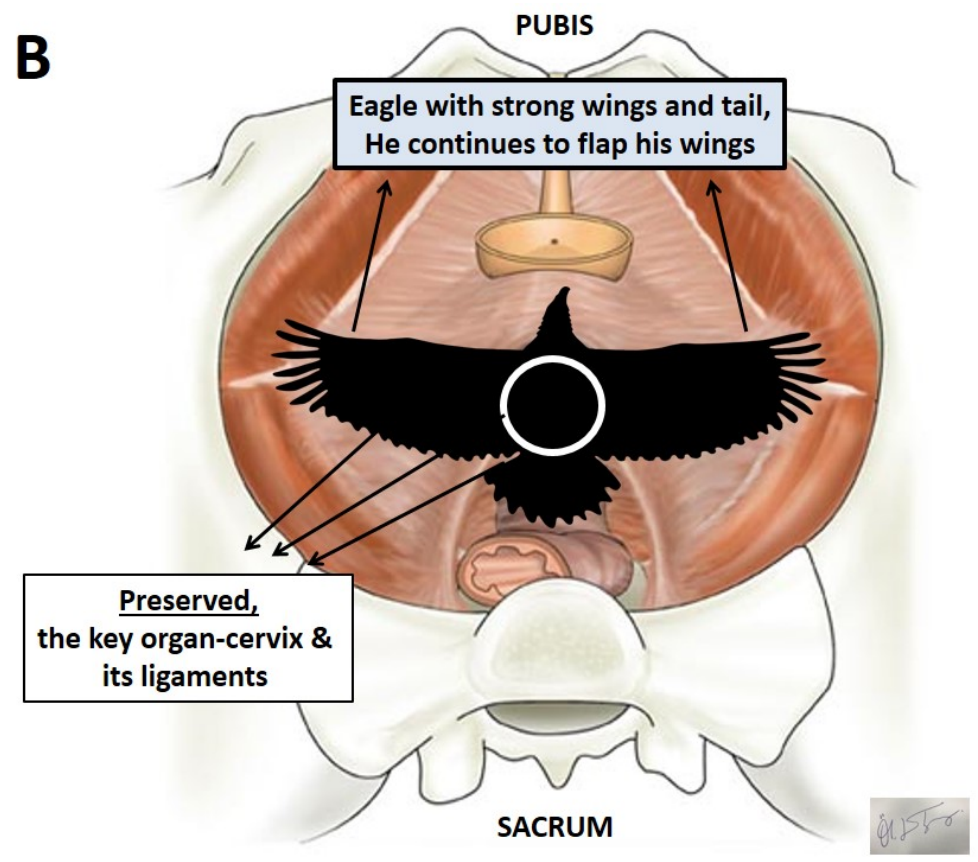

